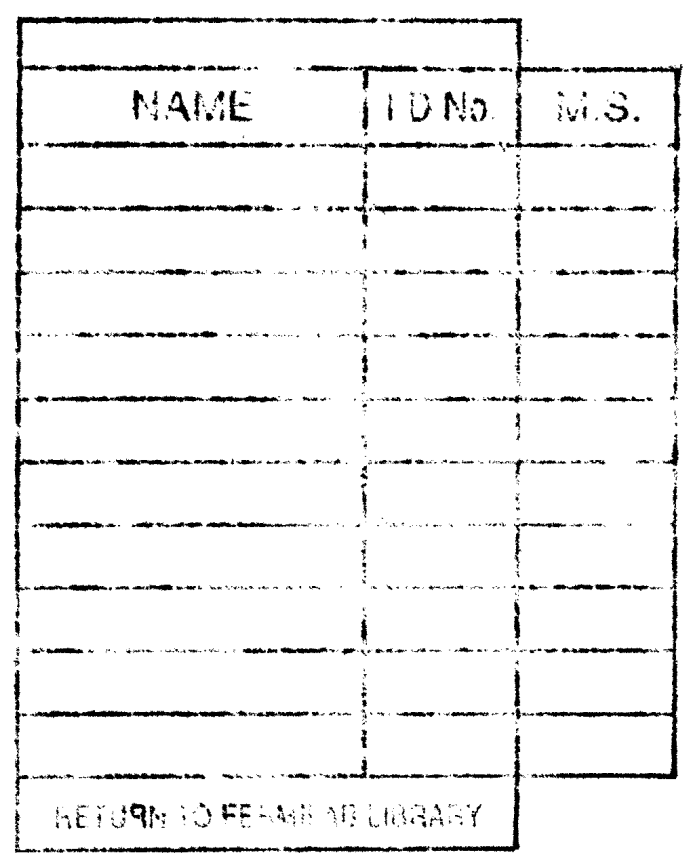

\title{
Clusters and Substructure Peculiar Velocities Central Galaxy Peculiar Velocities
}

Christina M. Bird

Department of Physics and Astronomy

University of Kansas, Lawrence, Kansas 66045 


\title{
SUBSTRUCTURE IN CLUSTERS AND CENTRAL GALAXY PECULIAR VELOCITIES
}

\author{
Christina M. Bird \\ Department of Physics and Astronomy \\ University of Kansas \\ Lawrence, KS 66045-2151 \\ Internet: tbird@kula.phsx.ukans.edu
}

\begin{abstract}
Formation theories for central dominant galaxies in clusters require them to be located at the minimum of the cluster gravitational potential. However, $32 \%$ ( 8 out of 25) of the clusters with more than 50 measured redshifts have central galaxies with significant velocity offsets (with respect to other cluster members). By studying their velocity distributions and correlations between velocity and position, I show that the presence of a large peculiar velocity is strongly correlated with the presence of substructure in these massive systems. About $85 \%$ (21 of 25) of all well-studied clusters show some evidence for substructure, in contrast to the $30-40 \%$ found when using only galaxy or gas distributions.

The correlation between substructure and central galaxy location verifies the hypothesis of Merritt (1985) and Tremaine (1990) that high peculiar velocities are indicative of recent merger events between less-massive systems of galaxies. Dynamical friction should act quickly to pull the central galaxy, the most massive discrete object in a cluster, to the minimum of the potential. The less-massive galaxies retain information about their primordial subclusters for a longer period of time.

I use an objective partitioning algorithm to assign cluster galaxies to their host subclumps. When galaxies are allocated in this fashion to their subclusters, $75 \%$ of the significant velocity offsets are eliminated. Only 2 out of the 25 clusters have central galaxies which are not centrally-located when substructure is considered in the analysis.
\end{abstract}

Accepted for publication in the Astronomical Journal

Subject headings: galaxies: clusters - galaxies: elliptical and lenticular, cD - galaxies: clustering 


\section{INTRODUCTION}

As Edwin Hubble pointed out in The Realm of the Nebulae, galaxies come in many shapes and sizes, or more accurately in many morphologies and masses. Ellipticals, spirals and irregulars can be found in every extragalactic environment, from low-density or field regions on up to densely-populated rich clusters. Only one type of galaxy seems to require the high-density environment. Giant elliptical galaxies are never found in the field. Because they appear to be preferentially located in the density maxima of clusters of galaxies (Beers \& Geller 1983), they are often referred to as central or central dominant galaxies.

The original definition of a central dominant galaxy (Mathews, Morgan \& Schmidt 1964) require the galaxy to be surrounded by an extended luminous envelope. Many authors have extended this definition to include any giant elliptical which is centrally located (cf. Morgan, Kayser \& White 1975; Albert, White \& Morgan 1977). Malumuth \& Kirshner (1985) found that central galaxies have a shallower surface brightness profile than ellipticals which are not brightest cluster members. Tremaine (1990) defines a central galaxy as a galaxy located at the minimum of the cluster potential well, which is itself identified using galaxy or gas density maxima. This is the definition I will adopt in this work.

Both optical and X-ray studies of luminosity functions suggest that central galaxies are not an extension of the "normal" cluster population of ellipticals (Malumuth \& Kirshner 1985; Morbey \& Morris 1983; Edge 1991), although they appear to lie close to the same fundamental plane which relates surface brightness, velocity dispersion, and radius (Oegerle \& Hoessel 1991). Measurements of the stellar velocity dispersions of central galaxies (and in some cases, their X-ray gas) indicate that they are the most massive discrete objects within clusters. For instance, Mould et al. (1990) use observations of the globular cluster system around M87, the centrally-located giant elliptical in the Virgo Cluster and find that its mass 
is $\sim 10^{13} \mathrm{M}_{\odot}$, much greater than that of typical ellipticals.

The origin of spiral and elliptical morphologies in "normal" cluster galaxies remains a mystery. The extreme properties of central galaxies suggest that their formation mechanisms must also be unusual, and probably involve their priveleged location within the cluster. Three mechanisms are generally considered likely for the growth of cDs within the cluster environment:

- In a relaxed cluster with a sufficiently high central gas density, intracluster gas will gradually cool and condense into the bottom of the potential well (Fabian, Nulsen \& Canizares 1984). This infall may trigger star formation in the central galaxy. Optical photometry and H- $\alpha$ imaging (McNamara \& O'Connell 1992) suggest that this is an unlikely formation mechanism for the nucleus of the galaxy, but the effect of the gas infall on the evolution of a centrally-located galaxy is likely to be important.

- Cluster member galaxies which pass through the center of the cluster may be tidally stripped, thereby providing material to be accreted onto a $\mathrm{cD}$. This may explain growth of the envelope, but cannot explain the central galaxy itself, since the velocity dispersion of cluster members is nearly three times that of stars in the $\mathrm{cD}$.

- The cD may form as the product of mergers, capture (as opposed to stripping) of less massive galaxies into the bottom of the potential well.

Distinguishing between these formation mechanisms probably requires both detailed models of the physical processes involved and careful distinction between classical "cD-type" galaxies (which are centrally-located and possess faint extended haloes), D galaxies (which are centrally-located but have normal surface brightness profiles) and giant ellipticals (which are not centrally located and have normal surface brightness profiles).

These formation models have been extensively studied both analytically and numerically 
(Merritt 1984, 1985; Richstone \& Malumuth 1983; Malumuth \& Richstone 1984; Malumuth 1992), under the important assumption that the central galaxies forms after the cluster virializes. Although evaluating them requires photometric and morphological distinctions which are beyond the scope of this dynamical study, they do share one important feature.

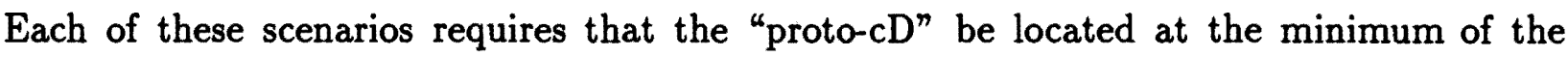
cluster gravitational potential for the formation process to occur sufficiently rapidly, which in turn implies that any peculiar velocity of the central galaxy relative to the cluster should be small. Despite this, several well-studied clusters have been cited as having significant $\mathrm{cD}$ velocity offsets (e.g. A2670, Sharples, Ellis \& Gray 1988; A1795, Hill et al. 1988; A85 and A2052, Malumuth et al. 1992; A2107, Oegerle \& Hill 1992). This poses a serious difficulty for the formation models. In particular, Malumuth (1992) showed that the formation of $\mathrm{cD}$ galaxies in mergers after cluster virialization could not reproduce the observed distribution of peculiar velocities in clusters.

Merritt (1985) and Tremaine (1990) suggest that, rather than forming after a rich system has collapsed, $\mathrm{cD}$ galaxies probably form before or during the virialization of rich clusters. Then the host clusters themselves merge and form an "evolutionary sequence" of morphologies: clusters with two dominant galaxies (Coma/A1656); systems with dumbbell galaxies (A400, A3266, A3391); and finally, rich relaxed clusters (A2029).. Optical and X-ray observations (Beers et al. 1991; Edge et al. 1992) also provide evidence for these hierarchical models of cluster formation. The hypothesis qualitatively explains many of the features of cD galaxies (see Tremaine 1990 for a summary), including the existence of a handful of systems with high peculiar velocities. Dynamical friction acts to pull a $\mathrm{cD}$ galaxy into the center of the gravitational potential of two merging clusters on a relatively short time-scale, compared to other galaxies in the system, because the mass of the central galaxy is so high. The remaining 
galaxies in the infalling cluster are much less massive, so their distribution in both position and velocity space should reflect their origin in a distinct subcluster even after the $\mathrm{cD}$ is at rest in the "new" potential. This may lead to a high value for the measured peculiar velocity (this was also pointed out by Hill et al. 1988), either because the substructure leads to an incorrect estimate of the velocity location, creating an apparent velocity offset, or because the central galaxy has not yet had time to settle into the potential minimum. The recent merger is detectable as substructure in the galaxy position and velocity distributions. For instance, ROSAT images of Coma suggest that the gE galaxy NGC 4839 in the southwestern clump is displaced from its subcluster's gas, which may be due to the galaxy's interaction with the massive central system (White et al. 1993).

The development of robust hypothesis tests for substructure in clusters (Dressler \& Shectman 1988; Bird \& Beers 1993; Bird 1993), when combined with the availibility of large cluster datasets in the literature, provides us with the first opportunity to test this hierarchical model of cluster formation. These tests are designed to quantify any structure in the velocity or galaxy distributions as well as correlations between velocity and position. By analyzing a cluster dataset using all available information, these diagnostics maximize the information we can obtain from a relatively small number of datapoints.

Deviations from Gaussian in a cluster's velocity distribution may imply anisotropic galaxy orbits and/or mixing of two or more distinct subpopulations of galaxies. They can be detected using a variety of techniques: those based on moments of the distribution (Fitchett \& Merritt 1988; Bird \& Beers 1993), those based on gaps (Bird \& Beers 1993) or those determined from a Gauss-Hermite expansion (Zabludoff et al. 1993). Computer simulations suggest that the classical coefficients of skewness and kurtosis are preferable as diagnostics of small deviations from Gaussian than the other techniques (Bird \& Beers 1993), and so 
they will be applied herein.

Well-formulated, powerful hypothesis tests for substructure in the galaxy distribution on the sky are more difficult to define than the 1-D diagnostics. The two-dimensional tests of mirror symmetry (the $\beta$-test), angular separation and density contrast (West et al. 1988) are shown by Bird (1993) to be inherently insensitive to the structures they claim to detect. Therefore the only 2-D test applied to the cluster dataset is the Lee statistic, introduced to the astronomical literature by Fitchett (1988). The Lee statistic quantifies the probability that two equal-sized groups provide a better description of the galaxy distribution than does a one-group fit.

Computer simulations of cluster formation and theoretical prejudice predict that a dynamically evolved cluster of galaxies will possess a roughly isothermal halo. This is the basis of the nearest neighbor tests for substructure, which are sensitive to local correlations between velocity and position. Although the centroid shift test (or $\alpha$-statistic, West \& Bothun 1990 ) is less sensitive than the cumulative deviation or $\Delta$-test of Dressler \& Shectman (1988b; also Bird 1993b), for consistency with other cluster analyses I apply both of them to the cluster dataset. In addition, I introduce the $\epsilon$-statistic in this context.

Of the 40 or so galaxy clusters with more than 50 measured redshifts (the minimum number required for a robust statistical analysis; Bird 1993), 25 have brightest cluster members of morphological type ' $\mathrm{gE}$,' ' $\mathrm{D}$ ' or ' $\mathrm{cD}$.' These systems are presented in Table 1 . To be included in the cluster dataset, a galaxy must have a velocity which is within $3 \mathrm{~S}_{B I}$ (defined below) of the average velocity of the system (this criterion defines the "naive" velocity filter given in column 3) and must lie within $1.5 h^{-1} \mathrm{Mpc}$ of the central galaxy. (Here I have taken $H_{0}=100 h \mathrm{~km} \mathrm{~s}^{-1} \mathrm{Mpc}^{-1}$.) The following quantities are listed in Table 1: column (1), the cluster name; column (2), the cluster right ascension and declination, found from the position 
of the central galaxy (see Beers \& Tonry 1986); (3) the velocity filter and number of galaxies meeting the membership criteria; (4) the recessional velocity, estimated with the biweight estimator of location $\mathrm{C}_{B I}$ (Beers, Flynn \& Gebhardt 1990), and the $90 \%$ confidence intervals about that value; (5) the velocity dispersion, determined using the biweight estimator of scale $\mathrm{S}_{B I}$ (Beers, Flynn \& Gebhardt 1990), and its associated $90 \%$ confidence intervals; (6) the line-of-sight velocity of the central galaxy and the error in that quantity, taken from the original source; (7) the source code. Positions and measurement uncertainties are taken from the original sources given in column 7 .

In Section 2, I review the robust method of assessing a central galaxy's location within a cluster (Gebhardt \& Beers 1991) and apply this technique to the cluster dataset. Hypothesis tests for substructure in clusters are presented in Section 3, and are used to study the clusters' velocity and galaxy distributions. In Section 4, I describe the objective partitioning algorithm and apply it to the cluster dataset. This work is summarized, conclusions are presented and future work described in Section 5.

\section{PECULIAR VELOCITIES OF CENTRAL GALAXIES}

Quintana \& Lawrie (1982) presented the first systematic study of peculiar velocities and concluded that central galaxies were preferentially (although not exclusively) located at the center of their host cluster's gravitational potential. Other recent studies include Sharples, Ellis \& Gray (1988), Zabludoff et al. (1990) and Malumuth et al. (1992). Each of these studies used slightly different definitions of the central galaxy offset velocity and evaluated significance in a non-robust manner, and concluded that a large fraction of clusters had central galaxies with significant peculiar velocities. But the results for different clusters are hard to compare to each other directly, because of the varying definitions. Gebhardt \& Beers (1991) point out that many claims of "speeding $\mathrm{cDs}$ " disappear when a rigorous statistical technique 
is applied to cluster datasets. In this section, I will review their physically-meaningful and statistically-robust measure of a central galaxy's peculiar velocity with respect to its host cluster. Intuitively, the physical idea is simple. Is the velocity of a cluster's central galaxy significantly displaced from the central location of the velocity distribution as a whole? In operation, the definition is a little more subtle.

In their studies of A1795 and A2670, Hill et al. (1988) and Sharples, Ellis \& Gray (1988) quantify the significance of a central galaxy's velocity by scaling it to measurement uncertainties in the location of the cluster velocity distribution, here called $\sigma_{m}$, and the uncertainty in the velocity of the central galaxy, $\sigma_{l}$ :

$$
Z_{T}=\frac{\delta v}{\left(\sigma_{l}^{2}+\sigma_{m}^{2}\right)^{1 / 2}}
$$

This definition is also adopted by Oegerle \& Hill (1992) and Hill \& Oegerle (1993) in their systematic studies of rich clusters, although these authors used the biweight estimator of scale $S_{B I}$ rather than the standard deviation $\sigma$ to estimate the uncertainty in the cluster mean velocity. Unfortunately, for non-Gaussian distributions, there is no simple analytical relationship between any estimator of the scale of the distribution and the uncertainty in its velocity location (cf. Beers, Flynn \& Gebhardt 1990). This may lead to an overestimate of the significance of a given peculiar velocity. About $40 \%$ of well-studied clusters have velocity distributions which are inconsistent with Gaussian (Bird 1993; this work), implying that use of the analytical relation between dispersion and uncertainty in the mean is hazardous at best. Even when the measured velocity distribution is consistent with Gaussian, the difference between bootstrapped confidence intervals (which consider sampling errors and uncertainties in the individual measurements themselves) and confidence intervals calculated analytically (assuming a Gaussian probability distribution for the sample distribution) can be as large as a factor of two (Beers, Flynn \& Gebhardt 1990; see also Mo, Jing \& Borner 
1992 for a discussion of the bootstrapping technique). Our ability to accurately measure the cluster average velocity should be one of the factors in deciding whether or not a central galaxy has a significant peculiar velocity (with respect to the rest of the cluster).

Additionally, from a physical point of view the issue is not: "Are the velocities consistent within the errors?" but rather: "Are the velocities consistent within $S_{B I}$ ?" because we are interested in the probability of drawing the $\mathrm{cD}$ velocity from a distribution with a dispersion $S_{B I}$. To be physically meaningful, the peculiar velocity should be scaled to the cluster velocity dispersion, $S_{B I}$.

Gebhardt \& Beers (1991) define a robust measure of the physical significance of a cD peculiar velocity:

$$
Z_{G B}=\frac{v_{C D}-C_{B I}}{S_{B I}}
$$

This quantity is hereafter called the " $Z$-score" for a cluster. The confidence intervals about this quantity are determined directly from the observational data, using a bootstrap resampling routine. To consider a velocity offset significant, the $90 \%$ confidence intervals of its $Z$-score should not bracket zero; this convention is chosen for consistency with the significance levels of the substructure diagnostics.

In Table 2 are presented the $Z$-scores for the 25 clusters in the database. Column (1) is the cluster name, (2) the peculiar velocity and (3) the $Z$-score. All velocities are given in $\mathrm{km} \mathrm{s}^{-1}$, and errors are taken from the original sources (listed in Table 1). In this table and hereafter, checkmarks after a cluster's name indicates that the cluster possesses a statistically-significant peculiar velocity.

$32 \%$ of the cD clusters ( 8 out of 25 ) have significant peculiar velocities. [A85 does not, even when the foreground group is excluded from the cluster membership, in contradiction to the conclusions of Malumuth et al. (1992). This is apparently due to the more rigorous 
definition of significance used herein, as first found by Gebhardt \& Beers (1990) using a smaller dataset.] This high fraction of significant velocity offsets is a major problem for all three formation scenarios. If this result is an indication that a third of all central galaxies are significantly displaced from the minimum of their cluster potential, then not only are formation theories in trouble, but tidal stripping should have shredded the low surface brightness envelopes in these systems (Merritt 1984).

Malumuth et al. (1992) propose that hierarchical clustering will produce a correlation between peculiar velocity and cluster richness, such that high peculiar velocities only occur in high velocity dispersion clusters. They claim this is because, in hierarchical models, the richest, most massive systems (on average) will have undergone the most recent merger event. This may not yet have been erased through dynamical evolution, and will be reflected in a large peculiar velocity. Additionally, in clusters which still retain distinct velocity populations, the global velocity dispersion is often over-estimated, which further reinforces this correlation.

In Figure 1, I present the Malumuth et al. (1992) correlation using the rich clusters from this study, as well as poor clusters with $\mathrm{cD}$ brightest cluster members. These are taken from an optical study by Beers et al. (1993). When the sample is extended over the largest statistically well-sampled range in velocity dispersions possible, the correlation that Malumuth et al. find is confirmed. The four crosses mark low-mass systems (MKW9, $N_{g a l}=$ 14; MKW10, $N_{g a l}=16 ;$ MKW3s, $N_{g a l}=10 ;$ MKW4s, $\left.N_{g a l}=17\right)$ from Beers et al. which appear to violate the relation. Even for the lower numbers of redshifts measured for the poor clusters, the use of bootstrapping to determine the confidence intervals about $S_{B I}$ and $Z$ makes the estimators statistically stable for most systems. In these cases, however, it is possible that the cluster is not evenly sampled, and that more data would reduce their 
peculiar velocities. If these points are excluded, Figure 1 certainly supports the hypothesis that high peculiar velocities are only found in high velocity dispersion systems.

\section{SUBSTRUCTURE DIAGNOSTICS}

In order to quantify the level of substructure present in a given cluster, we must first define what a cluster with no structure is. Theoretical and computational models of cluster formation predict that virialized systems should possess Maxwellian or near-Maxwellian velocity distributions (Saslaw et al. 1990; Ueda et al. 1993). Under this prediction, the line-of-sight velocity distributions observed for rich systems of galaxies are expected to be Gaussian (or perhaps, longer-tailed than Gaussian). Unfortunately, predictions for the distribution of galaxies or gas in projection on the sky are not as clear-cut. It does seem reasonable that a relaxed system should be single-peaked in number density, and spherically or elliptically symmetric. Finally, in a cluster with an isothermal dark matter halo, no correlation between position and velocity of the member galaxies is expected. In other words, if cluster galaxies exist within a common isothermal envelope of dark matter-the simplest assumption about cluster structure, and one which is consistent with current $\mathrm{X}$-ray observations and gravitational lensing experiments - the velocity dispersion is independent of radius within the cluster. Computer simulations suggest that tidal interactions between merging subclumps may drive the gravitational potential quickly toward isothermality (Roettinger Burns \& Loken 1993; Katz \& White 1993).

Therefore, the substructure diagnostics use the above predictions as definitions of their null hypotheses. To study deviations from Gaussian in the cluster velocity distributions, I have applied the coefficients of skewness and kurtosis (evaluated using the ROSTAT statistics

packages graciously provided by Timothy Beers). Skewness is a measure of the asymmetry 
of the distribution. For a dataset in which the central location and scale are not known $a$ priori, the skewness is defined as

$$
a_{3}=\frac{\sum_{i=1}^{N}\left(x_{i}-C_{\bar{x}}\right)^{3}}{N S_{x}^{3}}
$$

Here $\mathrm{C}_{\bar{x}}$ is the average value of the data, and $\mathrm{S}_{x}$ is their standard deviation.

The kurtosis quantifies the relative population of the tails of the dataset compared to its central region. The Gaussian is defined to be neutrally elongated. If a distribution is peakier than Gaussian and/or its tails are heavier than expected, it exhibits positive kurtosis, or is leptokurtic. In this case one might conclude that contamination by outliers, either interlopers in the system or data with unacceptably high errors, is a problem. A distribution which is less peaky than Gaussian and/or has light tails exhibits negative kurtosis, or is platykurtic. A boxy distribution may indicate that the system is multimodal, consisting of overlapping distinct populations.

The kurtosis is the fourth moment of the distribution about its mean:

$$
a_{4}=\frac{\sum_{i=1}^{N}\left(x_{i}-C_{\grave{x}}\right)^{4}}{N S_{x}^{4}}-3
$$

The factor of $S_{x}^{4}$ in the denominator makes the estimator scale-invariant. Evaluating the significance of the skewness and kurtosis is particularly simple. It is equal to the probability that a Gaussian distribution would have a value equal to that obtained for the dataset under consideration.

Fitchett (1988) introduced to the astronomical literature a maximum likelihood test of the galaxy distribution, called the Lee statistic or $L_{\text {rat }}$ (Lee 1979). The Lee statistic tests a cluster (or any 2-D dataset) for the presence of two equal-sized groups. Its great advantage over the 2-D diagnostics discussed in the Introduction is that it is a well-defined hypothesis 
test, with a null hypothesis of one group vs. the alternative of two groups being present in the data. Certainly many clusters appear to have bimodal galaxy distributions (Geller \& Beers 1982), so at first glance this seems to be a reasonable diagnostic. The algorithm for calculating $L_{r a t}$ is detailed and too long to be presented here, but may be found in Fitchett (1988) and Bird (1993).

To determine whether a value of $L_{r a t}$ is a rejection of the null hypothesis for a particular cluster, Monte Carlo simulations of random distributions are generated from the original datasets by scrambling the declinations. 500 simulations are performed for each cluster. The significance level is the probability that a random dataset will have as high a value of $L_{\text {rat }}$ as the observed cluster has.

The importance of local correlations between velocity and position was first exploited by Dressler \& Shectman (1988). Their cumulative deviation or $\Delta$-test measures the deviation from the global average velocity and global velocity dispersion of each individual galaxy in the cluster and its ten nearest neighbors:

$$
\Delta \equiv \sum_{i=1}^{N_{g a l}} \delta_{i}^{2}=\frac{11}{S_{x^{2}}(g l)^{2}} \sum_{i=1}^{N_{g a l}}\left[\left(C_{\bar{v}}(i)-C_{\bar{v}}(g l)\right)^{2}+\left(S_{v^{2}}(i)-S_{v^{2}}(g l)\right)^{2}\right]
$$

The subscript $g l$ refers to the kinematic quantity evaluated for the entire (or $g$ lobal) cluster dataset. The subscript $i$ refers to the quantity evaluated for the nearest neighbor group. $\Delta$ is normalized, under the assumption that the velocity dispersion profile is flat for the (spatial) region under study, by generating Monte Carlo realizations of the cluster and comparing their $\Delta$-values to that of the observed dataset.

Similarly, West \& Bothun (1990) define a test which measures the sensitivity of position centroid measurements to selection of galaxies by velocity range. The position of each galaxy in a given cluster dataset is weighted by the reciprocal of the velocity dispersion of its 10 
nearest neighbors in projection on the sky:

$$
w_{i}=\frac{1}{\sigma_{i}}
$$

Then for each galaxy $i$, the velocity-weighted position centroid is calculated for itself and its 10 nearest neighbors in velocity space:

$$
\begin{aligned}
& x_{c i}=\frac{\sum_{i=1}^{11} x_{i} w_{i}}{\sum_{i=1}^{11} w_{i}} \\
& y_{c i}=\frac{\sum_{i=1}^{11} y_{i} w_{i}}{\sum_{i=1}^{11} w_{i}}
\end{aligned}
$$

where the $w_{i}$ 's are the weights previously defined. Finally, the test statistic is calculated. The centroid shift is

$$
\alpha=\frac{1}{N_{g a l}} \sum_{i=1}^{N_{g a l}}\left[\left(x_{c}-x_{c i}\right)^{2}+\left(y_{c}-y_{c i}\right)^{2}\right]^{\frac{1}{2}}
$$

The unweighted $x$ - and $y$-centroids for the entire cluster dataset are designated by $x_{c}$ and $y_{c}$ respectively. The $\alpha$-statistic is normalized in the same fashion as the $\Delta$-statistic, through repeated Monte Carlo simulations.

Bird (1993, 1994) points out that using a constant value for the number of nearest neighbors reduces the sensitivity of these tests to significant structure. Silverman (1986) suggests that a value of

$$
N_{k e r n}=N_{g a l}^{\frac{1}{2}}
$$

maximizes sensitivity of the test to significant structure while reducing sensitivity to fluctuations which are within the Poisson noise. The appropriate value of $N_{\text {kern }}$ for each galaxy cluster is listed in Table 3 .

In both of these substructure diagnostics, the spatial and velocity dimensions of the cluster data are treated independently: the $\Delta$-test uses positions only to define nearest neighbor groups (hereafter NNGs), the $\alpha$-test uses the velocities only to select and weight 
galaxies for the position centroid calculation. But position and velocity data both retain information about the degree of dynamical processing in clusters. In order to exploit this fact, I define the $\epsilon$-statistic as

$$
\epsilon=\frac{1}{N_{g a l}} \sum_{i=1}^{N_{g a l}} M_{P M E}
$$

where $M_{P M E}$ is the projected mass estimator (Bahcall \& Tremaine 1981; Heisler, Tremaine \& Bahcall 1985) calculated for each of the NNGs.

$$
M_{P M E}=\xi\left(\frac{24}{\pi G N_{k e r n}}\right) \sum_{j=1}^{N_{k e r n}} v_{z j}^{2} r_{j}
$$

The constant $\xi$ depends on the configuration of test particle orbits. It is here taken to be $\frac{4}{3}$, the value for isotropic orbits. $v_{z j}$ is the peculiar velocity of a galaxy with respect to the NNG average velocity, $C_{B I}(i)$, and $r_{j}$ is its projected distance from the NNG center. The galaxy $i$ for which the NNG is selected does not contribute to $M_{P M E}$, because it is by definition located at the center of the NNG and its projected separation is therefore zero.

The $\epsilon$-statistic is normalized in the same way as the $\Delta$-test, by using the original cluster dataset. Random realizations of a cluster with an identical velocity histogram are obtained by shuffling the velocities and positions of the observed cluster galaxies. This Monte Carlo technique effectively removes any structure in the original data. It provides an empirical estimate of the average mass per NNG including Poisson fluctuations in a cluster with a flat velocity dispersion profile.

A detailed comparison of the three nearest neighbor diagnostics, using computer simulations to study the power and sensitivity of the tests, is presented in Bird $(1993,1994)$. Study of Table 3 reveals that the 3 diagnostics do not consistently return the same answers for a given dataset. For instance, A2063 has a marginally significant value of $\Delta$ but a nonsignificant $\epsilon$ result. Computer simulations show that although the $\epsilon$-statistic is more sensitive 
to plane-of-the-sky structures than $\Delta$, the combination of velocity and position data introduces a larger source of scatter in the Poisson values generated during its normalization. This decreases its sensitivity. In other words, because the Poisson fluctuations for the $\epsilon$-test are larger than for the $\Delta$-test, it is not as sensitive a diagnostic. Nonetheless, it does sometimes provide evidence for structure that the other diagnostics miss. The centroid shift test suffers from even larger Poisson fluctuations than does $\epsilon$. Bird (1994) shows that its convoluted algorithm of weights and filters makes it the least sensitive of the nearest neighbor tests.

In Table 3, I have summarized the results of the substructure diagnostics for the clusters with high central galaxy peculiar velocity. I require a significance level of less than $10 \%$ for a rejection of the null hypothesis with any of these statistics. These values are given in the columns to the right of the cluster name.

In contradiction to the commonly-held belief that clusters with central galaxies have smooth and relaxed morphologies, these systems have an extremely high frequency of substructure, as Table 4 demonstrates. "Low- $Z$ " systems do not have a significant velocity offset; "high- $Z$ " systems do. The table summarizes the frequency of substructure detections for each statistic ( 0.000 means that no clusters have that sort of structure; 1.000 means they all do). I have excluded the Lee statistic in the following discussion because it has no sensitivity to velocity structure. Since we are trying to correlate substructure in clusters with a velocity-related property (their $Z$-score), it makes sense at least at first to consider only diagnostics which are themselves dependent on (or related to) velocity. The Lee statistic, at least as applied herein, is sensitive only to structure on the sky. A merger occurring in the plane of the sky is probably taking place between two subclusters at similar recessional velocities. In such systems, no matter how recent the merger the central galaxy will always appear to be centrally-located, i.e. will have a low $Z$-score. Table 3 shows that in fact low- $Z$ 
systems do frequently have a positive Lee statistic.

The high- $Z$ clusters consistently demonstrate a higher frequency of substructure than do their low- $Z$ counterparts. In particular, all of the NNG diagnostics reveal that clusters with a high peculiar velocity are significantly more likely to reject the null hypothesis of a relaxed system than those with no large velocity offset. Also important is the result that the only clusters with no indications of substructure (A1809, A2124, and A2199) have low velocity offsets. These trends all lend support to the hypothesis of Merritt $(1984,1985)$, Hill et al. 1988, Tremaine (1990) and Malumuth (1992) that central galaxies form in host groups which then collapse and virialize to become rich, relaxed clusters. Although this idea has been in the literature for nearly a decade, it has required the compilation of a large dataset of well-studied clusters and the development of rigorous statistical techniques presented in this work to demonstrate that the predicted trend exists in the data.

In the past, $30-40 \%$ of all clusters have been found to demonstrate substructure (Geller \& Beers 1982; Jones \& Forman 1984), although Baier (1983) claimed a much higher fraction. These surveys have been based on tracer particle distributions (of galaxies and gas, respectively) and have therefore been insensitive to substructure existing in velocity distributions, that is, along our line of sight. The frequencies of rejection of the Gaussian hypothesis for velocity distributions (also between $30-40 \%$, see Table 4 ) imply that the fraction of unrelaxed clusters may be more than twice as high, $\sim 85 \%$. Taken another way, only $\sim 15 \%$ ( 3 out of 25) of the well-studied clusters in this database show no indication of substructure in either their velocity or position distributions. If the cluster sample is extended to include clusters such as A2151 which do not have a central dominant galaxy (Bird 1993), the fraction of clusters with no detectable substructure drops to $\sim 8 \%$. This substructure may not be dynamically important in every cluster, but it is certainly sufficient to lend an added degree 
of uncertainty to any kinematic or dynamical analyses of clusters.

\section{CORRECTING FOR SUBSTRUCTURE}

Many authors working on the problem of "speeding central galaxies" have proposed substructure as a solution (i.e., Sharples, Ellis \& Gray 1988; Gebhardt \& Beers 1991; Malumuth et al. 1992). In particular, identifying the most appropriate membership for the determination of peculiar velocities was the primary goal of the Malumuth et al. (1992) subjective membership criteria. They identify cluster members on the basis of their position within the cluster wedge diagrams and velocity histograms, also making use of cluster diagnostics such as those described in Section 3. They admit that this method does not provide a quantitative estimate of correctness or confidence in their assignments, but they claim that these criteria are more likely to provide the "correct" cluster membership than the blind application of a $3 \sigma$ clip (Yahil \& Vidal 1977) based on velocities alone.

Having clearly established the relationship between large offset velocities and substructure, even in clusters with prominent central galaxies, our next goal is similarly to identify the most appropriate host system for the central galaxy in the cluster datasets. To minimize the subjectivity of the analysis, I have chosen to allocate galaxies to subclusters using an objective partitioning algorithm called KMM (McLachlan \& Basford 1987; Adams, McLachlan \& Basford 1993). KMM is a maximum-likelihood algorithm which assigns objects into groups and and assesses the improvement in fitting a multi-group model over a single group model. KMM can be applied to data of any dimensionality. It has already been applied to one dimensional astronomical datasets, such as the color/metallicity distributions of extragalactic globular cluster systems (Ashman \& Zepf 1993; Zepf \& Ashman 1993; Ostrov et al. 1993; Lee \& Geisler 1993). Ashman, Bird \& Zepf (1994) present an analysis of the statistical behavior of the algorithm and its use as a tool to detect bimodality in one-dimensional 
datasets. It has been applied to the velocity and position datasets of galaxy clusters to identify substructure (A2670, Bird 1994; A548, Davis et al. 1994) as the basis of detailed dynamical analyses.

The KMM algorithm fits a user-specified number of Gaussian distributions to a dataset and assesses the improvement of that fit over a single Gaussian. KMM is a hypothesis test of the null hypothesis that the data is consistent with a unimodal Gaussian probability distribution. But in addition, it provides the maximum-likelihood estimate of the unknown $n$-mode Gaussian and an assignment of objects into groups, unlike the more familiar $\chi^{2}$ or KS tests. This fitting procedure requires as input a first guess at either the assignments of the objects into groups or the vector means and covariance matrices of the model being fit, as well as the measurements on the objects to be partitioned. KMM is most appropriate in situations where theoretical and/or empirical arguments indicate that a Gaussian model is reasonable. This is probably valid in the case of cluster velocity distributions, where gravitational interactions drive the system toward Gaussian. It is less likely to be appropriate for the projected positions of galaxies on the sky. But Beers \& Tonry (1986) find that for any individual cluster of galaxies, the difference in surface density profile between a Gaussian fit and a de Vaucouleur fit is not large.

The likelihood ratio test statistic (hereafter LRTS) provided by KMM is an estimate of the improvement in going from a 1 -mode to a $g$-mode fit. The significance of any particular LRTS may be estimated by using a $\chi^{2}$ distribution, at least in the univariate homoscedastic case, or preferably by using a resampling technique such as the bootstrap (McLachlan 1987). This level is the fraction of times that as high a LRTS would be calculated if the dataset was generated under the null hypothesis of a unimodal parent distribution. That is, the significance level for any particular KMM run is the chance that the routine has incorrectly 
identified a unimodal distribution as $g$-modal.

Selecting the best-fit model is based on the significance level of the fit, the algorithm's estimate of the correct allocation rate (how confident the algorithm is that any individual object has been assigned to the proper group) and the stability of the means and dispersions of the Gaussians being fit. Because the three dimensional diagnostics seem to be the most sensitive indicators of the presence of substructure, I have chosen to simultaneously fit the velocity and position data for each of the clusters. I have fit two, three and four groups to the datasets. Few of them contain enough points to make a fit of more than four groups believable. In cases where the LRTS is equal for different models, I have arbitrarily selected the model with the lowest number of groups to be the best fit.

It is important to emphasize that the problem of estimating the number of underlying populations in a sample dataset is underspecified. Therefore the best-fit models are not mathematically unique. Subjectivity is introduced into the use of KMM because the best-fit number of groups is not unique or specified a priori. Nonetheless, the algorithm does allow the user to allocate the data into groups in a statistically consistent way, which one hopes is more objective than the membership criteria which have been used in the past. In addition, use of the KMM algorithm does provide quantitative measures of membership confidence and goodness of fit, removing some (although not all) of the subjectivity from the substructure analysis.

The details of the KMM models, selection of the best-fit partition and dynamical analyses based on those models are given in Bird (1993, the high- $Z$ clusters; 1994, A2670). To save space I have not tabulated the results of each analysis here. The reader interested in specifically how a best-fit model was chosen for any particular galaxy cluster is refered to Bird (1993). In Table 5, I present for each of the clusters the preferred number of groups, 
$\mathrm{N}_{g r}$, and the number of galaxies in the group to which the central galaxy is assigned; the location $\mathrm{C}_{B I}$ of the central galaxy's host subcluster and the $90 \%$ confidence intervals about that value; its dispersion $S_{B I}$ and the associated confidence intervals; the peculiar velocity of the central galaxy with respect to its host subsystem; and finally the recalculated $Z$-score and its $90 \%$ confidence intervals.

Note that for clusters with independent determinations of substructure (such as Pinkney et al. 1993 and Malumuth et al. 1992), KMM has objectively identified the same structures found "by eye" and through use of the Dressler-Shectman statistic. This provides some reassurance that despite the uncertainties in the algorithm, in most cases KMM is allocating galaxies to subclusters in a reasonable way, or at least in a way which is consistent with previous work.

Examination of Table 5 reveals that the low- $Z$ systems all retain their low peculiar velocities after substructure allocation, although in the case of A2199 that may not be strictly true (Gregory \& Thompson 1984 point out that A2199 is gravitationally bound to A2197 and therefore, perturbation of the central galaxy from the center of its host cluster is perhaps unsurprising). Of the eight high- $Z$ systems, only two (A1736 and A3558), retain their high offset velocities after substructure is "corrected" using the objective partitioning technique. To verify that these high offsets are not an artifact of an unsatisfactory model of the data, I have re-partitioned the original datasets following each of the KMM models. In all of them, the central galaxy remains significantly displaced from the minimum of its host system's potential well. It is possible that the displacement is physically-meaningful, although in both of these cases the total number of datapoints is quite low. More redshifts and positions will presumably clarify their dynamical situation.

After KMM analysis, only 3 galaxy clusters (A85, A426 and A2199) are found to consist 
of only 1 group. Two of the systems with no detectable substructure (A1809 and A2124) are classified as bimodal systems, although in A1809 only 7 galaxies are excluded from the central subcluster. In all, 9 clusters are bimodal, 7 have three subclusters and 6 have 4 . These numbers are somewhat tentative, given the subjectivity involved in determining the best-fit model for some cases, but the high frequency of multimodality is consistent with the high frequency of substructure found in Section 3.

A careful comparison of the velocity dispersions before and after substructure allocation reveals that occasionally $S_{B I}$ is larger after the partition is applied. This is somewhat counter-intuitive, since in systems in which the merger is primarily along the line-of-sight, overlapping velocity distributions can result in an over-estimate of the dispersion of the system as a whole. If the KMM best-fit models are adequate descriptions of the physical structure in the clusters, this result suggests that partitions based primarily on structure in a velocity histogram may be misleading, or may not make the most efficient use of the information available. On the other hand, it is no doubt true that KMM has made mistakes in some of its allocations of member galaxies, and that the "Occam's razor" approach to choosing a best-fit model will sometimes cause mis-assignments. Nonetheless, in cases where the best-fit models can be independently verified, either by comparison with X-ray observations or with other subjective substructure allocations (for instance, A2634, Pinkney et al. 1993; Davis et al. 1994), KMM appears to be providing reliable information.

Having determined the corrected distribution of kinematic and dynamical properties of galaxy clusters when substructure is included in the analysis, it is instructive to compare the observations to the previously-cited theoretical models of cluster formation. In a forthcoming paper, we will use these dynamical properties for a revision of the optical and X-ray correlations. Here, we can compare the distribution of observed peculiar velocities to those 
predicted by Malumuth's (1992) simulations. First of all, note that although his simulated clusters each have a velocity dispersion of $400 \mathrm{~km} \mathrm{~s}^{-1}$, the clusters in his observed distribution range from $\sim 500 \mathrm{~km} \mathrm{~s}^{-1}$ for the poor cluster MKW4 to $1400 \mathrm{~km} \mathrm{~s}^{-1}$ for A2052. The large scatter in velocity dispersion may be responsible for the excess of high peculiar velocities when compared to the simulations. Therefore I normalize the peculiar velocities to the velocity dispersions before the comparison; that is, I plot the $Z$-score rather than the peculiar velocity. This obviously does not change the shape of the distribution derived from the simulations but does skew the observed distribution toward smaller values, making even the uncorrected distribution more consistent with the theoretical prediction than the original analysis implied.

Unlike the original Malumuth figures, in which large deviations from the theoretical predictions exist for each bin of the histogram, proper scaling of the peculiar velocity distribution reduces the inconsistency even without correction for substructure, as shown in Figure 2. Using the correctly-allocated host subclusters, the theoretical and observed predictions are inconsistent at only a $2 \sigma$ level. Despite the prevalence of substructure in rich clusters, perhaps this result implies that central galaxy formation after cluster virialization is still a viable formation mechanism. At least it is not as strongly eliminated from consideration as Malumuth (1992) maintains.

\section{DISCUSSION AND SUMMARY}

The combination of relatively large datasets and robust hypothesis tests helps to clarify the relationship between central galaxy peculiar velocities and dynamical evolution in clusters. Clusters with centrally-located brightest cluster members have a relatively low frequency of velocity substructure. They more often (35\% of the time, 9 of 25 ) possess 
quantifiable structure in their position distributions, which suggests that at least some of them have formed through "plane-of-the-sky" mergers between less massive systems. These clusters are presumably at similar recessional velocities; deconvolving overlapping distributions with the same means is extremely difficult, and the one-dimensional diagnostics are not likely to detect this sort of structure. The $\Delta$ - and $\alpha$-statistics rarely detect structure in low- $Z$ clusters. The $\epsilon$-statistic is positive for them about $40 \%$ of the time ( 7 out of 17 ). This is because its dependence on velocity and position makes it somewhat sensitive to plane-of-the-sky structure, unlike the other NNG diagnostics (see also Bird 1993b).

High- $Z$ clusters are much likelier to exhibit structure in their velocity distributions than their low-velocity counterparts. They never (or hardly ever) possess positive Lee statistics, and have a high frequency of detections with the NNG techniques. As already seen in the dynamical analysis of A2670 (Bird 1994), these results are consistent with the merger of subclusters primarily along our line of sight. Unfortunately, this orientation makes independent verification of these subclusters difficult, since X-ray images will not be able to resolve structure in this orientation.

The substructure analysis confirms the hypothesis of Merritt (1985), Tremaine (1990) and Malumuth (1992) that high peculiar velocities are an indication of recent merger events. The substructure diagnostics are hypothesis tests of the data's consistency with what we believe a relaxed system should look like. Their rejections of the null hypotheses for the high- $Z$ systems, therefore, support the interpretation that high velocity offsets are themselves an indication of dynamical turbulence.

It has generally been assumed that highly clumpy, irregular galaxy clusters have formed recently. However, some computer simulations suggest that substructure may survive for a significant fraction of a Hubble time, forming quasi-stable structures as the cluster virializes 
(cf. bimodal or "dumb-bell" structures; Cavaliere \& Colafrancesco 1990). If this is true, then the existence of substructure no longer implies that a cluster has formed recently. The high mass of central galaxies implies that dynamical friction will force them to a cluster's potential minimum on a timescale of a third of a Hubble time or so (Tremaine 1990). This is rather quicker than the timescale for less massive galaxies to reach equilibrium within the potential well. The correlation between peculiar velocity and substructure implies that the mergers must have occurred relatively recently, thereby strengthening the assumption that the substructure diagnostics are reliable hypothesis tests of a cluster's dynamical state.

This is not to imply that the substructure tests alone are sufficient to completely determine the dynamics of a cluster of galaxies. Small number statistics even in the largest cluster catalogs introduce an appreciable source of uncertainty, even when sophisticated statistical techniques are used. The importance of reliable X-ray temperature determinations in merging systems cannot be over-emphasized, as an independent measurement of the depth of the gravitational potential.

The prevalence of substructure in clusters with central galaxies resolves the apparent conflict between formation theories for these ultraluminous galaxies and the high fraction of clusters with displaced central galaxies. When substructure is corrected by assigning central galaxies to their host subclusters, 6 of the 8 significant peculiar velocities disappear. This result does not favor any of the formation theories summarized in the Introduction, although it may provide some difficulty for the cooling flow model. Edge et al. (1992) suggest that merger events between subclusters of comparable sizes disrupt the quasi-equilibrium conditions necessary for a cooling flow. These disruptions are relatively short, however, and may not have a major effect on the amount of mass deposited over a Hubble time.

Tremaine (1990) proposes that clusters with double-nuclei central galaxies (the "dumb- 
bell galaxies" defined by Mathews, Morgan \& Schmidt 1964) may be a profitable subject for this sort of analysis. In a hierarchical clustering sequence, these galaxies are formed during the rapid merger of two galaxies which are themselves centrally-located within two merging subclusters. Because the merging timescale for the two central galaxies is extremely short, dumb-bell galaxies may be a clear signature of dynamical youth. In that case, application of the statistical diagnostics and partitioning algorithm contained in this work will provide clear evidence for the interacting subclusters, as we have already seen to be the case in clusters with classical central galaxies. This link between dumb-bell evolution and largerscale substructure is evident in the Beers et al. study of A400 (1992). Mergers between central galaxies may also promote the formation of wide-angle tail radio sources, as seen in A2634 by Pinkney et al. (1993). It may be possible, for sufficiently large cluster datasets, to associate the individual dumb-bell components with their host subclusters.

I am very grateful to Eliot Malumuth and Bill Oegerle for playing devil's advocate and Timothy Beers for software and a firm grounding in statistical methodology. Keith Ashman, Scott Tremaine and the Canadian Institute for Theoretical Astrophysics provided support and encouragement during this project. John Hill, Ray Sharples, Jason Pinkney and Alan Dressler provided computer versions of their cluster catalogs, for which I am appreciative. John Hill improved the text through his careful and prompt referee'ing. This work was completed in partial fulfillment of the requirements for the Ph.D program at the University of Minnesota, and supported in part by the Department of Physics and Astronomy, Michigan State University, and by NSF grant OSR-9255223 at the University of Kansas. 


\section{REFERENCES}

Adams, P., McLachlan, G. \& Basford, K. 1993, private communication

Albert, C.E., White, R.A. \& Morgan, W.W. 1977, ApJ, 211, 309

Ashman, K.M., Bird, C.M. \& Zepf, S.E. 1994, in preparation

Ashman, K.M. \& Zepf, S.E. 1993, in Globular Clusters in the Context of their Parent

Galaxies, edited by J. Brodie and G. Smith (Dordrecht: Kluwer), p. 776

Bahcall, J.N. \& Tremaine, S. 1981, ApJ, 244, 805

Baier, F.W. 1983, Astron. Nacht., 5, 211

Beers, T.C., Flynn, K. \& Gebhardt, K. 1990, AJ, 100, 32

Beers, T.C., Forman, W., Huchra, J.P., Jones, C. \& Gebhardt, K. 1991, AJ, 102, 1581

Beers, T.C. \& Geller, M.J. 1983, ApJ, 274, 491

Beers, T.C., Huchra, J.P., Kriessler, J. \& Bird, C.M. 1994, in preparation

Beers, T.C., Gebhardt, K., Huchra, J.P., Forman, W. \& Jones, C. 1992, ApJ, 400, 410

Beers, T.C. \& Tonry, J.L. 1986, ApJ, 300, 557

Bird, C.M. 1993, Ph.D thesis, University of Minnesota and Michigan State University

Bird, C.M. 1994a, AJ, in preparation

Bird, C.M. 1994b, ApJ, to appear 20 February

Bird, C.M. \& Beers, T.C. 1993, AJ, 105, 1586

Cavaliere, A. \& Colafrancesco, S. 1990, in Clusters of Galaxies, ed. W.R. Oegerle, M. J.

Fitchett, and L. Danly, (New York: Cambridge University Press), pg. 43

Chapman, G., Huchra, J.P. \& Geller, M. 1988, CfA preprint

Davis, D.S., Bird, C.M., Mushotzky, R.F. \& Odewahn, S.C. 1994, ApJ, submitted

Dressler, A. 1980, ApJ, 236, 351

Dressler, A. \& Shectman, S. 1988a, AJ, 95, 284 
Dressler, A. \& Shectman, S. 1988b, AJ, 95, 985

Edge, A.C. 1991, MNRAS, 250, 103

Edge, A.C., Stewart, G.C. \& Fabian, A.C. 1992, MNRAS, 258, 177

Fabian, A.C., Nulsen, P.E.J. \& Canizares, C.R. 1984, Nature, 310, 733

Fabricant, D., Kurtz, M., Geller, M., Zabludoff, A., Mack, P. \& Wegner, G. 1993, AJ, 105, 788

Fitchett, M.J. 1988, MNRAS, 230, 169

Fitchett, M. \& Merritt, D. 1988, ApJ, 335, 18

Gebhardt, K. \& Beers, T.C. 1991, ApJ, 383, 72

Geller, M. \& Beers, T.C. 1982, PASP, 94, 421

Giovanelli, R. \& Haynes, M. 1985, ApJ, 292, 404

Gregory, S.A. \& Thompson, L.A. 1984, ApJ, 286, 422

Guzman, R., Lucey, J.R., Carter, D. \& Terlevich, R.J. 1992, MNRAS, 257, 187

Heisler, J., Tremaine, S. \& Bahcall, J.N. 1985, ApJ, 298, 8

Hill, J.M. \& Oegerle, W.R. 1993, AJ, 106, 831

Hill, J.M., Hintzen, P., Oegerle, W.R., Romanishin, W., Lesser, M.P., Eisenhamer, J.D. \& Batuski, D.J. 1988, ApJ, 332, L23

Hubble, E. 1936, The Realm of the Nebulae, (New Haven: Yale University Press)

Jones, C. \& Forman, W. 1984, ApJ, 276, 38

Katz, N. \& White, S.D.M. 1993, ApJ, 412, 455

Kent, S.M. \& Sargent, W.L.W. 1983, AJ, 88, 697

Lee, K.L. 1979, JAmStat, 74, 708

Lee, M.G. \& Geisler, D. 1993, AJ, 106, 493

Malumuth, E.M. 1992, ApJ, 386, 420 
Malumuth, E.M. \& Kirshner, R.P. 1985, ApJ, 291, 8

Malumuth, E.M. \& Richstone, D.O. 1984, ApJ, 276, 413

Malumuth, E.M., Kriss, G.A., Dixon, W.V.D., Ferguson, H.C. \& Ritchie, C. 1992, AJ, 104, 495

Mathews, T.A., Morgan, W.W. \& Schmidt, M. 1964, ApJ, 140, 35

McLachlan, G.J. 1987, Appl. Statist., 36, 318

McLachlan, G.J. \& Basford, K.E. 1988, Mixture Models, (New York: Marcel Dekker)

McNamara, R.B. \& O'Connell, R.W. 1992, ApJ, 393, 579

Merritt, D. 1984, ApJ, 276, 26

Merritt, D. 1985, ApJ, 289, 18

Mo, H.J., Jing, Y.P. \& Borner, G. 1992, ApJ, 392, 452

Morbey, C. \& Morris, S. 1983, ApJ, 274, 502

Morgan, W.W., Kayser, S. \& White, R.A. 1975, ApJ, 199, 545

Mould, J.R., Oke, J.B., de Zeeuw, P.T. \& Nemec, J.M. 1990, AJ, 99, 1823

Oegerle, W.R. \& Hill, J.M. 1992, AJ, 104, 2078

Oegerle, W.R. \& Hoessel, J.G. 1991, ApJ, 375, 15

Ostrov, P., Geisler, D. \& Forte, J.C. 1993, AJ, 105, 1762

Pinkney, J., Rhee, G., Burns, J.O., Hill, J.M., Oegerle, W., Batuski, D. \& Hintzen, P. 1993, ApJ, 416, 36

Quintana, J. \& Lawrie, 1982, AJ, 87, 1

Richstone, D.O. \& Malumuth, E.M. 1983, ApJ, 268, 30

Richter, O.G. 1987, A\&ASuppl, 77, 237

Roettinger, K., Burns, J.O. \& Loken, C. 1993, ApJLett, 407, L53

Saslaw, W.C., Chitre, S.M., Itoh, M. \& Inagaki, S. 1990, ApJ, 365, 419 
Sharples, R., Ellis, R. \& Gray, P. 1988, MNRAS, 231, 479

Sodre, L., Capelato, H.V., Steiner, J.E., Proust, D. \& Mazure, A. 1992, MNRAS, 259, 233

Teague, P.F., Carter, D. \& Gray, P.M. 1990, ApJSuppl, 72, 715

Tremaine, S. 1990, in Dynamics and Interactions of Galaxies, ed. R. Wielen, (Berlin: SpringerVerlag), pg. 394

Ueda, H., Itoh, M. \& Suto, Y. 1993, ApJ, 408, 3

West, M.J. \& Bothun, G.D. 1990, ApJ, 350, 36

West, M.J., Oemler, A. \& Dekel, A. 1988, ApJ, 327, 1

White, S.D.M., Briel, U.G. \& Henry, J.P. 1993, MNRAS, 261, L8

Whitmore, B.C. \& Gilmore, D. 1991, ApJ, 367, 64

Yahil, A. \& Vidal, N.V. 1977, ApJ, 214, 347

Zabludoff, A.I., Huchra, J.P. \& Geller, M.J. 1990, ApJSuppl, 74, 1

Zabludoff, A.I., Franx, M. \& Geller, M.J. 1993, CfA preprint 


\section{FIGURE CAPTIONS}

Figure 1: The velocity offset of central dominant galaxies is plotted against their velocity dispersion $S_{B I}$. Stars indicate rich clusters from this study; solid circles indicate poor clusters from Beers et al (1993). The crosses are the 4 poor clusters with high $Z$-scores and low velocity dispersions, discussed in the text. Error bars on $S_{B I}$ are the $90 \%$ confidence intervals given in Table 1.

Figure 2: The observed distributions of peculiar velocities in rich clusters of galaxies, before and after correction for substructure, to be compared with Malumuth (1992) Figure 4 and binned in the same increments. The upper panels are the original histograms; the lower panels provide a "double-root residual" transformation of the difference between the observed distributions and Malumuth's simulations (see Gebhardt \& Beers 1991; Ashman, Bird \& Zepf 1994 for a detailed description of the double root residual). Note that even without substructure correction, deviations from the predicted distribution of peculiar velocities are all within $3 \sigma$. When substructure is considered in the analysis, only the second bin of the histogram deviates by $2 \sigma$ from the predicted value. 
TABLE 1

cD Clusters with 50 or more Measured Redshifts

\begin{tabular}{|c|c|c|c|c|c|c|}
\hline Cluster & $\begin{array}{l}\text { R.A. } \\
\text { Decl. }\end{array}$ & $\begin{array}{l}\text { Vel. Filter } \\
\mathbf{N}_{\text {gal }}\end{array}$ & $\begin{array}{c}\mathrm{C}_{B I} \\
\text { (confidence interval) }\end{array}$ & $\begin{array}{c}\mathrm{S}_{B I} \\
\text { (confidence interval) }\end{array}$ & $\begin{array}{c}\text { cD Velocity } \\
\epsilon\end{array}$ & Source \\
\hline \multirow[t]{2}{*}{$\mathrm{A} 85^{\mathrm{a}}$} & 003918.6 & $13899-19493$ & 16708 & 810 & 16647 & 1 \\
\hline & -093438 & 109 & $(-134,134)$ & $(730,886)$ & 126 & \\
\hline \multirow[t]{2}{*}{ A119 } & 005342.7 & $10512-16118$ & 13324 & 862 & 13246 & 12 \\
\hline & -013133 & 79 & $(-172,148)$ & $(722,1027)$ & 45 & \\
\hline \multirow[t]{2}{*}{ A193 } & 012507.6 & $11948-17143$ & 14559 & 726 & 14466 & 2 \\
\hline & 084158.2 & 56 & $(-172,166)$ & $(618,856)$ & 65 & \\
\hline \multirow[t]{2}{*}{ A194 } & 012311.5 & $2849-7884$ & 5288 & 530 & 5417 & 9 \\
\hline & -013825 & 83 & $(-92,80)$ & $(423,679)$ & 20 & \\
\hline \multirow[t]{2}{*}{ A399 } & 025753.2 & $17851-25822$ & 21571 & 1183 & 21378 & 2 \\
\hline & 130152 & 108 & $(-189,197)$ & $(1075,1309)$ & 38 & \\
\hline \multirow[t]{2}{*}{ A401 } & 025857.9 & $17851-25822$ & 22090 & 1141 & 22275 & 2 \\
\hline & 133459.1 & 122 & $(-176,164)$ & $(1040,1273)$ & 58 & \\
\hline \multirow[t]{2}{*}{ A426 } & 031628.8 & $1541-9335$ & 5451 & 1262 & 5316 & 10 \\
\hline & 411954 & 155 & $(-158,178)$ & $(1130,1433)$ & 78 & \\
\hline \multirow[t]{2}{*}{ A496 } & 043118.8 & $7334-12414$ & 9865 & 741 & 9811 & 1 \\
\hline & -132153 & 138 & $(-107,102)$ & $(658,837)$ & 42 & \\
\hline \multirow[t]{2}{*}{ A754 } & 090653.1 & $13676-18840$ & 16231 & 719 & 16317 & 3 \\
\hline & -092941 & 69 & $(-137,132)$ & $(609,862)$ & 45 & \\
\hline \multirow[t]{2}{*}{ A1060 } & 103421.6 & $1654-5730$ & 3697 & 630 & 3725 & 11 \\
\hline & -271605 & 132 & $(-93,90)$ & $(574,696)$ & 19 & \\
\hline \multirow[t]{2}{*}{ A1644 } & 125433.2 & $10955-17355$ & 14164 & 919 & 14239 & 3 \\
\hline & -170822 & 90 & $(-141,152)$ & $(805,1075)$ & 45 & \\
\hline A1736 & 132402.6 & $10619-17005$ & 13795 & 955 & 13450 & 3 \\
\hline (Back) & -265305 & 60 & $(-206,237)$ & $(841,1062)$ & 45 & \\
\hline \multirow[t]{2}{*}{ A1795 } & 134852.5 & $15827-21995$ & 18881 & 834 & 18972 & 2 \\
\hline & 263535 & 82 & $(-139,149)$ & $(715,976)$ & 18 & \\
\hline
\end{tabular}


TABLE 1 continued

\begin{tabular}{|c|c|c|c|c|c|c|}
\hline Cluster & $\begin{array}{l}\text { R.A. } \\
\text { Decl. }\end{array}$ & $\begin{array}{l}\text { Vel. Filter } \\
\mathbf{N}_{\boldsymbol{g a l}}\end{array}$ & $\begin{array}{c}\mathrm{C}_{B I} \\
\text { (confidence interval) }\end{array}$ & $\begin{array}{c}\mathrm{S}_{B I} \\
\text { (confidence interval) }\end{array}$ & $\begin{array}{c}\text { cD Velocity } \\
\epsilon\end{array}$ & Source \\
\hline \multirow[t]{2}{*}{ A1809 } & 135306.5 & 20884-26496 & 23679 & 782 & 23632 & 2 \\
\hline & 050859 & 50 & $(-172,208)$ & $(657,930)$ & 16 & \\
\hline \multirow{2}{*}{ A1983 } & 145035.2 & $10853-15994$ & 13464 & 646 & 13295 & 3 \\
\hline & 165414 & 79 & $(-194,117)$ & $(517,830)$ & 45 & \\
\hline \multirow[t]{2}{*}{ A2052 } & 151416.9 & $5877-16001$ & 10732 & 1404 & 10310 & 1 \\
\hline & 071216 & 80 & $(-252,503)$ & $(1056,1805)$ & 25 & \\
\hline \multirow[t]{2}{*}{$A 2063^{b}$} & 152305.3 & 7963-13099 & 10531 & 827 & 10320 & 2 \\
\hline & 083632 & 56 & $(-224,197)$ & $(708,975)$ & 28 & \\
\hline \multirow[t]{2}{*}{ A2107 } & 153939.1 & $10103-14568$ & 12337 & 684 & 12615 & 4 \\
\hline & 214656.8 & 68 & $(-132,141)$ & $(580,810)$ & 86 & \\
\hline \multirow[t]{2}{*}{ A2124 } & 154459.1 & $16319-23060$ & 19663 & 872 & 19810 & 2 \\
\hline & 360633 & 62 & $(-197,169)$ & $(758,1023)$ & 30 & \\
\hline \multirow[t]{2}{*}{ A2199 } & 162655.6 & $6614-11925$ & 9190 & 829 & 9473 & 5 \\
\hline & 393938 & 65 & $(-114,138)$ & $(711,953)$ & 150 & \\
\hline \multirow[t]{2}{*}{ A2634 } & 233829.4 & $5215-13832$ & 9409 & 1077 & 9154 & 6 \\
\hline & 270151.6 & 177 & $(-112,122)$ & $(925,1289)$ & 59 & \\
\hline \multirow[t]{2}{*}{ A2670 } & 235139.6 & $18886-26714$ & 22848 & 1037 & 23282 & 7 \\
\hline & -104151.1 & 230 & $(-165,185)$ & $(956,1146)$ & 100 & \\
\hline \multirow[t]{2}{*}{ A3558 } & 132508.0 & $11132-17460$ & 14306 & 923 & 14037 & 8 \\
\hline & -311343 & 80 & $(-167,195)$ & $(821,1043)$ & 21 & \\
\hline \multirow[t]{2}{*}{ DC0107-46 } & 010742.4 & $4028-10904$ & 7421 & 1032 & 7237 & 3 \\
\hline & -461150 & 108 & $(-86,79)$ & $(924,1157)$ & 62 & \\
\hline \multirow[t]{2}{*}{$\mathrm{DC} 1842-63$} & 184234.6 & $2501-6604$ & 4482 & 522 & 4472 & 3 \\
\hline & -632304 & 92 & $(4396,4561)$ & $(440,621)$ & 32 & \\
\hline
\end{tabular}


Notes to TABLE 1

All velocities given in this table are measured in $\mathrm{km} \mathrm{s}^{-1}$.

Source List:

$1=$ Malumuth et al. 1992

$2=$ Hill \& Oegerle 1993

$3=$ Dressler \& Shectman 1988

$4=$ Oegerle \& Hill 1992

$5=$ Gregory \& Thompson

$6=$ Pinkney et al. 1993

7 = Sharples, Ellis \& Grey 1988

$8=$ Teague et al. 1990

$9=$ Chapman et al. 1988

$10=$ Kent \& Sargent 1983

$11=$ Richter et al. 1989

12 = Fabricant et al. 1993

a 11 galaxies within the velocity filter and the radial cutoff are excluded from the final dataset. This is based on the partitioning of Malamuth et al. (1992) and Beers et al. (1991), who concluded that the galaxies between $13,000 \mathrm{~km} \mathrm{~s}^{-1}$ and $15,000 \mathrm{~km} \mathrm{~s}^{-1}$ were foreground to the main cluster. Removing these galaxies affects only the result of the $\epsilon$-test, and not the significance of the peculiar velocity.

$b_{5}$ galaxies within the velocity filter and the radial cutoff are excluded from the final dataset. They appear to be contamination from a background sheet of galaxies at $\sim 13,600 \mathrm{~km} \mathrm{~s}^{-1}$. Including them in the substructure analysis does not change the results, because there are so few of them and because they have no spatial clumpiness. 
TABLE 2

$\mathrm{Z}_{G B}$ for Rich Clusters

\begin{tabular}{|c|c|c|}
\hline Cluster & $\mathrm{V}_{\text {pec }}\left(\mathrm{km} \mathrm{s}^{-1}\right)$ & $\mathrm{Z}_{G B}$ \\
\hline A85 & -250 & $-0.307(-0.595,0.016)$ \\
\hline A119 & -74 & $-0.085(-0.300,0.132)$ \\
\hline A193 & -91 & $-0.123(-0.407,0.166)$ \\
\hline$A 194 \sqrt{ }$ & 129 & $0.242(0.066,0.436)$ \\
\hline A399 & -182 & $-0.153(-0.324,0.025)$ \\
\hline A401 & 173 & $0.151(-0.043,0.325)$ \\
\hline A426 & -137 & $-0.108(-0.299,0.051)$ \\
\hline A496 & -53 & $-0.007(-0.236,0.103)$ \\
\hline A754 & 84 & $0.115(-0.106,0.339)$ \\
\hline A1060 & 29 & $0.045(-0.115,0.208)$ \\
\hline A1644 & 70 & $0.008(-0.119,0.277)$ \\
\hline A1736 $\sqrt{ }$ & -337 & $-0.350(-0.593,-0.076)$ \\
\hline A1795 & 84 & $0.100(-0.090,0.299)$ \\
\hline A1809 & -46 & $-0.058(-0.307,0.232)$ \\
\hline A1983 & -164 & $-0.251(-0.574,0.066)$ \\
\hline A2052 $\sqrt{ }$ & -447 & $-0.312(-0.561,-0.131)$ \\
\hline A2063 $\sqrt{ }$ & -210 & $-0.251(-0.502,-0.016)$ \\
\hline A2107 $\sqrt{ }$ & 273 & $0.396(0.008,0.698)$ \\
\hline A2124 & 140 & $0.159(-0.080,0.390)$ \\
\hline A2199 & 279 & $0.334(-0.001,0.750)$ \\
\hline A2634 $\sqrt{ }$ & -252 & $-0.233(-0.378,-0.009)$ \\
\hline A2670 $\sqrt{ }$ & 411 & $0.394(0.194,0.599)$ \\
\hline A3558 $\sqrt{ }$ & -261 & $-0.281(-0.520,-0.009)$ \\
\hline DC0107-46 & -184 & $-0.177(-0.387,0.003)$ \\
\hline DC1842-63 & -13 & $-0.030(-0.214,0.189)$ \\
\hline
\end{tabular}


TABLE 3

Substructure Results for Clusters

\begin{tabular}{|c|c|c|c|c|c|c|}
\hline Cluster & Skew & Kurt & Lee & $\Delta$ & $\alpha$ & $\epsilon$ \\
\hline A85 & 0.425 & 0.021 & 0.980 & 0.032 & 0.362 & 0.410 \\
\hline A119 & 0.254 & 0.280 & 0.638 & $<0.001$ & 0.418 & $<0.001$ \\
\hline A193 & 0.116 & 0.420 & 0.390 & 0.188 & 0.090 & 0.362 \\
\hline A194 $\sqrt{ }$ & $<0.001$ & $<0.001$ & 0.374 & 0.032 & 0.492 & 0.084 \\
\hline A399 & 0.365 & 0.014 & $<0.001$ & 0.456 & 0.016 & 0.322 \\
\hline A401 & 0.230 & 0.177 & $<0.001$ & 0.502 & 0.050 & 0.218 \\
\hline A426 & 0.108 & 0.215 & 0.276 & 0.106 & 0.386 & 0.048 \\
\hline A496 & 0.103 & 0.132 & 0.098 & 0.514 & 0.160 & 0.366 \\
\hline A754 & 0.449 & 0.168 & 0.548 & 0.436 & 0.028 & $<0.001$ \\
\hline $\mathrm{A} 1060$ & 0.331 & 0.118 & 0.030 & $<0.001$ & 0.014 & $<0.001$ \\
\hline A1644 & 0.029 & 0.204 & 0.854 & 0.132 & 0.034 & 0.050 \\
\hline$A 1736 \sqrt{ }$ & 0.464 & 0.006 & 0.894 & 0.028 & 0.050 & $<0.001$ \\
\hline A1795 & 0.059 & 0.081 & 0.166 & 0.390 & 0.426 & 0.056 \\
\hline A1809 & 0.252 & 0.352 & 0.224 & 0.448 & 0.540 & 0.146 \\
\hline A1983 & $<0.001$ & 0.035 & 0.026 & 0.266 & 0.246 & 0.244 \\
\hline$A 2052 \sqrt{ }$ & 0.004 & 0.201 & 0.788 & $<0.001$ & 0.024 & 0.002 \\
\hline$A 2063 \sqrt{ }$ & 0.241 & 0.504 & 0.468 & 0.088 & 0.482 & 0.358 \\
\hline$A 2107 \sqrt{ }$ & 0.481 & 0.589 & 0.156 & $<0.001$ & 0.044 & 0.058 \\
\hline A2124 & 0.348 & 0.412 & 0.438 & 0.468 & 0.288 & 0.132 \\
\hline A2199 & 0.356 & 0.281 & 0.642 & 0.312 & 0.172 & 0.128 \\
\hline$A 2634 \sqrt{ }$ & 0.002 & 0.004 & 0.756 & $<0.001$ & 0.568 & 0.404 \\
\hline A2670 $\sqrt{ }$ & 0.006 & 0.139 & 0.238 & $<0.001$ & 0.044 & 0.054 \\
\hline A3558 $\sqrt{ }$ & 0.187 & 0.132 & 0.458 & 0.028 & 0.408 & 0.196 \\
\hline DC0107-46 & 0.071 & 0.114 & 0.078 & 0.028 & 0.222 & 0.070 \\
\hline DC1842-63 & 0.028 & 0.148 & 0.380 & 0.100 & 0.110 & 0.510 \\
\hline
\end{tabular}


TABLE 4

Summary of Substructure Results

\begin{tabular}{clll}
\hline Test & All Clusters & High $Z_{G B}$ & Low $Z_{G B}$ \\
\hline Skewness & 0.360 & 0.500 & 0.294 \\
Kurtosis & 0.280 & 0.375 & 0.235 \\
Lee & 0.240 & 0.000 & 0.353 \\
$\Delta$ & 0.480 & 1.000 & 0.235 \\
$\alpha$ & 0.400 & 0.500 & 0.353 \\
$\epsilon$ & 0.480 & 0.625 & 0.412 \\
\hline \hline
\end{tabular}


TABLE 5

$Z_{G B}$ Corrected for Substructure

\begin{tabular}{|c|c|c|c|c|c|}
\hline Cluster & $\begin{array}{l}\mathrm{N}_{g r} \\
\mathrm{~N}_{g a l}\end{array}$ & $\begin{array}{c}\mathrm{C}_{B I} \\
\text { (confidence interval) }\end{array}$ & $\begin{array}{c}\mathrm{S}_{B I} \\
\text { (confidence interval) }\end{array}$ & $v_{p e c}$ & $\begin{array}{c}Z \\
\text { (confidence interval) }\end{array}$ \\
\hline \multirow[t]{2}{*}{ A85 } & 1 & 16708 & 810 & -250 & -0.307 \\
\hline & 109 & $(-134,134)$ & $(730,886)$ & & $(-0.595,0.016)$ \\
\hline \multirow[t]{2}{*}{ A119 } & 2 & 13002 & 1036 & 246 & 0.244 \\
\hline & 35 & $(-350,274)$ & $(815,1250)$ & & $(-0.072,0.569)$ \\
\hline \multirow[t]{2}{*}{ A193 } & 4 & 14551 & 515 & -95 & -0.185 \\
\hline & 16 & $(-192,246)$ & $(362,691)$ & & $(-0.745,0.415)$ \\
\hline \multirow[t]{2}{*}{ A194 } & 3 & 5328 & 470 & 90 & 0.189 \\
\hline & 70 & $(-141,148)$ & $(392,568)^{\circ}$ & & $(-0.040,0.396)$ \\
\hline \multirow[t]{2}{*}{ A399 } & 2 & 21455 & 1224 & -73 & -0.060 \\
\hline & 88 & $(-212,225)$ & $(1108,1355)$ & & $(-0.249,0.141)$ \\
\hline \multirow[t]{2}{*}{ A401 } & 4 & 22179 & 785 & 92 & 0.116 \\
\hline & 69 & $(-156,161)$ & $(704,896)$ & & $(-0.145,0.354)$ \\
\hline \multirow[t]{2}{*}{ A426 } & 1 & 5451 & 1262 & -137 & -0.108 \\
\hline & 155 & $(-158,178)$ & $(1130,1433)$ & & $(-0.299,0.051)$ \\
\hline \multirow[t]{2}{*}{ A496 } & 3 & 9685 & 533 & 124 & 0.231 \\
\hline & 84 & $(-85,107)$ & $(457,619)$ & & $(-0.001,0.475)$ \\
\hline \multirow[t]{2}{*}{ A754 } & 3 & 16368 & 1079 & -48 & -0.045 \\
\hline & 23 & $(-457,349)$ & $(836,1313)$ & & $(-0.489,0.406)$ \\
\hline \multirow[t]{2}{*}{$\mathrm{A} 1060$} & 2 & 3742 & 710 & -17 & -0.023 \\
\hline & 69 & $(-134,153)$ & $(632,788)$ & & $(-0.246,0.203)$ \\
\hline \multirow[t]{2}{*}{ A1644 } & 2 & 14238 & 921 & 3 & 0.003 \\
\hline & 83 & $(-149,154)$ & $(780,1089)$ & & $(-0.198,0.190)$ \\
\hline \multirow[t]{2}{*}{ A1736 $\sqrt{ }$} & 4 & 12878 & 528 & 588 & 1.114 \\
\hline & 17 & $(-280,275)$ & $(441,664)$ & & $(0.523,1.853)$ \\
\hline \multirow[t]{2}{*}{ A1795 } & 2 & 18868 & 912 & 98 & 0.106 \\
\hline & 58 & $(-185,204)$ & $(783,1104)$ & & $(-0.129,0.337)$ \\
\hline
\end{tabular}


TABLE 5 continued

\begin{tabular}{|c|c|c|c|c|c|}
\hline Cluster & $\begin{array}{l}\mathbf{N}_{g r} \\
\mathbf{N}_{g a l}\end{array}$ & $\begin{array}{c}\mathrm{C}_{B I} \\
\text { (confidence interval) }\end{array}$ & $\begin{array}{c}\mathrm{S}_{B I} \\
\text { (confidence interval) }\end{array}$ & $\mathbf{v}_{\text {pec }}$ & $\begin{array}{c}Z \\
\text { (confidence interval ) }\end{array}$ \\
\hline \multirow[t]{2}{*}{ A1809 } & 2 & 23639 & 851 & -8 & -0.009 \\
\hline & 43 & $(-272,230)$ & $(697,993)$ & & $(-0.330,0.357)$ \\
\hline \multirow[t]{2}{*}{ A1983 } & 2 & 13349 & 721 & -52 & -0.072 \\
\hline & 24 & $(-350,310)$ & $(462,1093)$ & & $(-0.885,0.367)$ \\
\hline \multirow[t]{2}{*}{ A2052 } & 3 & 10331 & 714 & -22 & -0.030 \\
\hline & 40 & $(-176,197)$ & $(566,857)$ & & $(-0.282,0.296)$ \\
\hline \multirow[t]{2}{*}{ A2063 } & 3 & 10251 & 706 & 67 & 0.096 \\
\hline & 38 & $(-210,187)$ & $(597,823)$ & & $(-0.221,0.435)$ \\
\hline \multirow[t]{2}{*}{ A2107 } & 4 & 12567 & $\mathbf{5 7 7}$ & 42 & 0.070 \\
\hline & 30 & $(-174,167)$ & $(450,754)$ & & $(-0.331,0.582)$ \\
\hline \multirow[t]{2}{*}{ A2124 } & 2 & 19649 & 906 & 159 & 0.173 \\
\hline & 33 & $(-269,269)$ & $(760,1041)$ & & $(-0.187,0.505)$ \\
\hline \multirow[t]{2}{*}{ A2199 } & 1 & 9190 & 829 & 279 & 0.334 \\
\hline & 65 & $(-114,138)$ & $(711,953)$ & & $(-0.001,0.750)$ \\
\hline \multirow[t]{2}{*}{ A2634 } & 3 & 9254 & 824 & -102 & -0.122 \\
\hline & 109 & $(-123,134)$ & $(691,967)$ & & $(-0.301,0.084)$ \\
\hline \multirow[t]{2}{*}{ A2670 } & 4 & 23231 & 251 & 49 & 0.191 \\
\hline & 76 & $(-44,49)$ & $(227,276)$ & & $(-0.595,0.930)$ \\
\hline \multirow[t]{2}{*}{ A3558 $\sqrt{ }$} & 3 & 14305 & 781 & -262 & -0.333 \\
\hline & 57 & $(-187,178)$ & $(683,892)$ & & $(-0.579,-0.088)$ \\
\hline \multirow[t]{2}{*}{ DC0107-46 } & 2 & 7389 & 1034 & -153 & -0.147 \\
\hline & 96 & $(-178,202)$ & $(919,1164)$ & & $(-0.346,0.053)$ \\
\hline \multirow[t]{2}{*}{ DC1842-63 } & 4 & 4350 & 565 & 125 & 0.216 \\
\hline & 24 & $(-188,222)$ & $(448,703)$ & & $(-0.216,0.709)$ \\
\hline
\end{tabular}




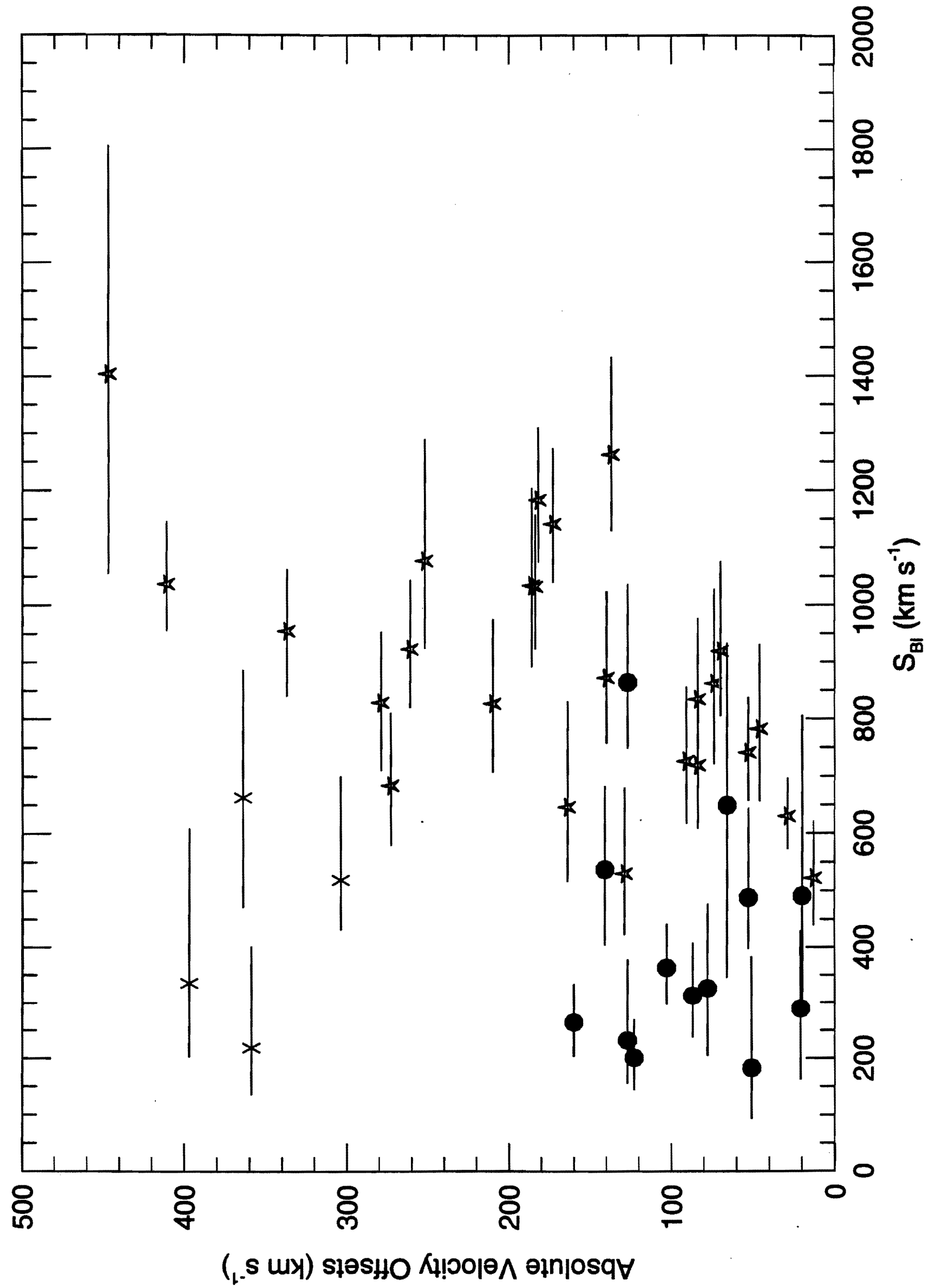

ईै 
No Correction for Substructure
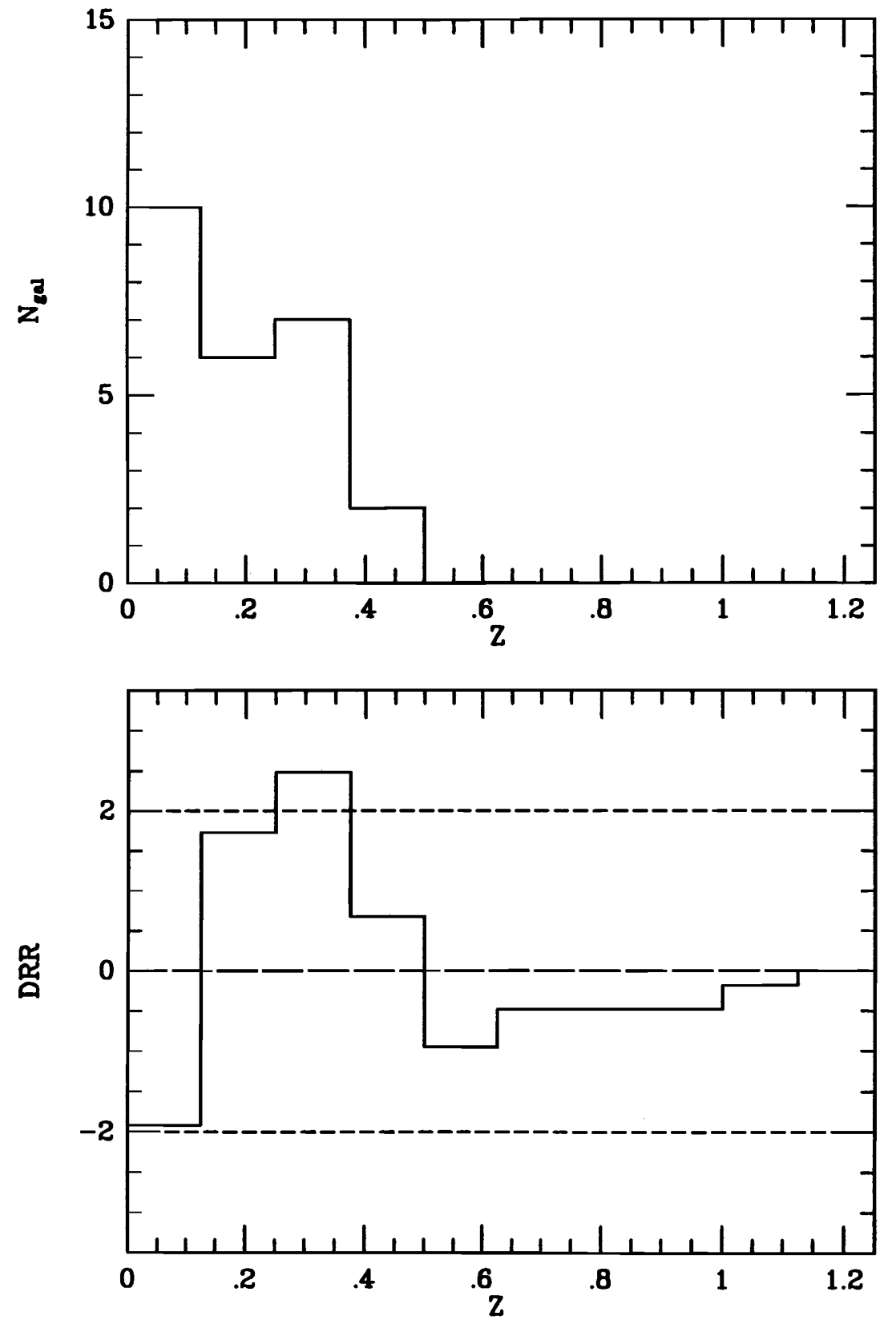

Corrected for Substructure
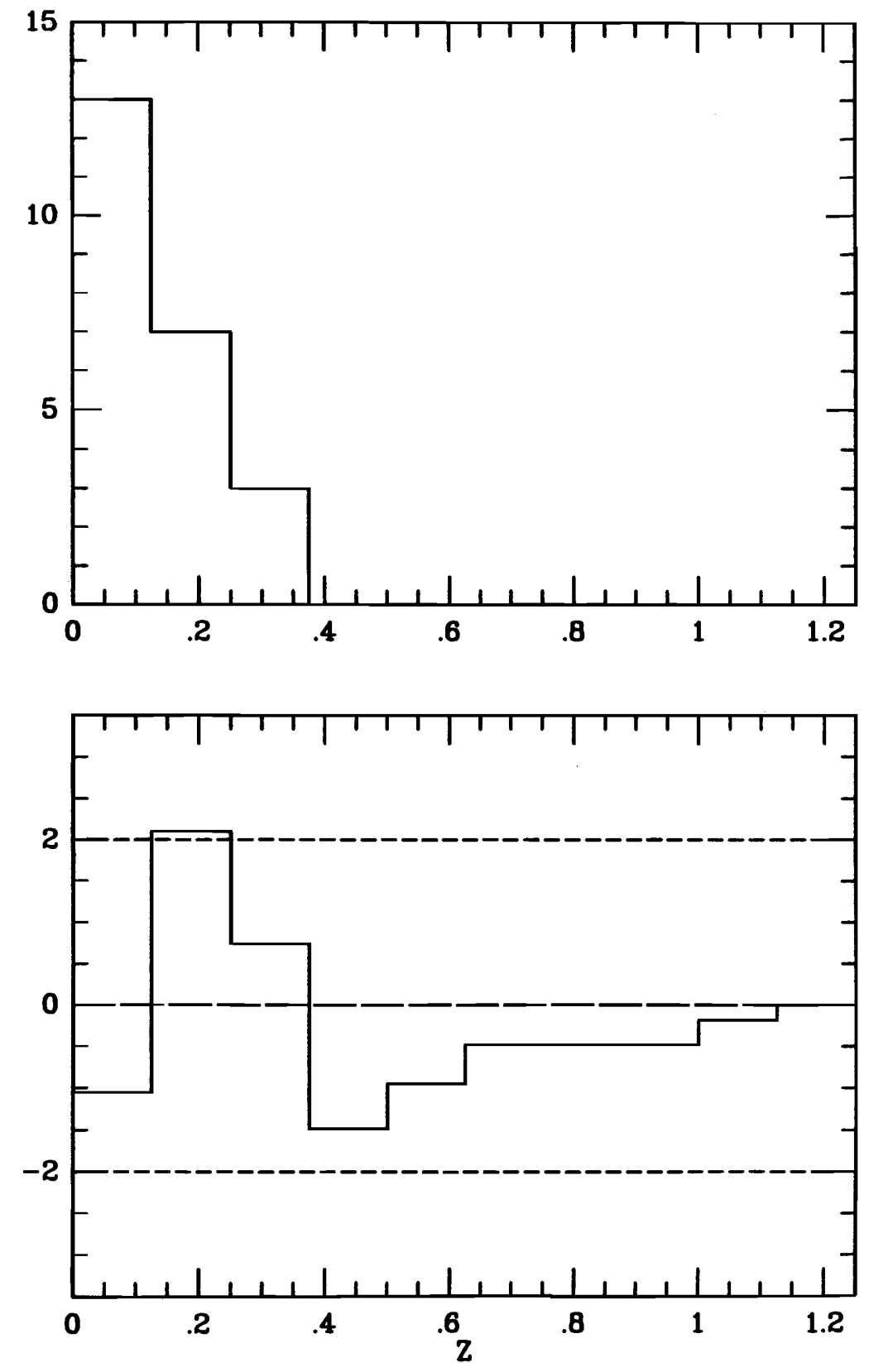

Figure 2 